\title{
Surfactant-free syntheses and pair distribution function analysis of osmium nanoparticles
}

Mikkel Juelsholt, ${ }^{a, \ddagger}$ Jonathan Quinson, ${ }^{a, \neq, \neq}$ Emil T. S. Kjær, ${ }^{a}$ Baiyu Wang, ${ }^{a}$ Rebecca

Pittkowski, ${ }^{a}$ Susan R. Cooper, ${ }^{a}$ Tiffany. L. Kinnibrugh, ${ }^{b}$ Søren B. Simonsen, ${ }^{c}$ Luise Theil Kuhn, ${ }^{c}$ María Escudero-Escribano ${ }^{a}$ and Kirsten M. Ø. Jensen ${ }^{a, *}$

a. Department of Chemistry, University of Copenhagen, 5 Universitetsparken, Copenhagen, 2100, Denmark

b. X-ray Science Division, Advanced Photon Source, Argonne National Laboratory, Argonne, 9700 S Cass Ave, Lemont, IL 60439, USA

c. Department of Energy Conversion and Storage, Technical, University of

Denmark, Fysikvej Bldg. 310, Lyngby, DK-2800 Kgs., Denmark

* jonathan.quinson@chem.ku.dk, kirsten@chem.ku.dk

$\ddagger$ Equal contributors 


\section{Abstract}

A surfactant-free synthesis of precious metal nanoparticles performed in alkaline low boiling point solvents has been recently reported. Mono-alcohols are here investigated as solvent and reducing agents to obtain colloidal Os nanoparticles by this low temperature $\left(<100^{\circ} \mathrm{C}\right)$ surfactant-free synthesis. The effect of precursor $\left(\mathrm{OsCl}_{3}\right.$ or $\mathrm{H}_{2} \mathrm{OsCl}$ ), precursor concentration (up to $100 \mathrm{mM}$ ), solvent (methanol or ethanol), presence or absence of base $(\mathrm{NaOH})$ and addition of water (0 to $100 \mathrm{v} . \%)$ on the resulting nanomaterials is discussed. It is fond that no base is required to obtain Os nanoparticles as opposed to the case of Pt or Ir NPs. The robustness of the synthesis for concentration of precursor up to $100 \mathrm{mM}$ allows to perform X-ray total scattering with pair distribution function (PDF) analysis that shows that the 1-2 nm hcp NPs forms from chain-like $\left[\mathrm{OsO}_{x} \mathrm{Cly}\right]$-complexes .

\section{Keywords}

Nanoparticles; Osmium; Pair Distribution Function; Surfactant-free; Synthesis 


\section{Findings}

Precious metals are limited resources yet fundamental for a range of applications such as medicine or catalysis [1-3]. There are only few reports on Osmium (Os) $[4,5]$, see Figure $\mathbf{S} 1$ in supporting information $(\mathrm{SI})$, partly due to Os scarcity and because the highly toxic $\mathrm{OsO}_{4}$ compound is easily formed. However, Os and Osbased complexes and nanomaterials have been reported [6-9] and studied for their catalytic [10], optical and medical properties [11-17], in experimental and theoretical work [18].

Common wet-chemical syntheses require surfactants, viscous solvents or shape directing agents $[5,17,19-21]$ that act as ligands to stabilize colloidal nanoparticles (NPs). These additives can bring impurities, can be toxic and add cost to the synthesis. Surfactant-free syntheses therefore bear promising features for fundamental research but also industrial scale production [22-24]. A surfactant-free synthesis method to produce Pt, Ir, Ru or bimetallic NPs has been reported [22]. It only requires a mono-alcohol as solvent and reducing agent [25] a base and a metal precursor to obtain size controlled NPs [26, 27]. This approach leads to catalysts that are more active than those prepared for example in polyols $[28,29]$. Here we investigate if this simple synthetic approach using mono-alcohols is suitable for the synthesis of Os NPs.

The synthesis of Os NPs is here easily performed in closed containers made of polypropylene as described in the SI. As opposed to a classical reflux set up using glassware [22] or to the use of microwaves [30], visible or UV light [31], this allows for a rapid screening of a number of experimental parameters for long 
synthesis times, e.g. 6 hours at moderate temperature, e.g. $90^{\circ} \mathrm{C}$. Studies of NP synthesis have made it clear that a number of seemingly simple experimental parameters can play a significant role for nanomaterial synthesis [5, 32]. We therefore here screen the influence of several experimental parameters across a large parametric space by investigating the influence of the precursor: $\mathrm{OsCl}_{3}$ and $\mathrm{H}_{2} \mathrm{OsCl}_{6}$; the nature of solvent: methanol and ethanol; the absence or presence of a base $(\mathrm{NaOH})$ and the effect of adding water: $0,10,25,50,66,75$ or 100 v.\%. Table S1 gives an overview of the different parameters investigated for this parametric study. Figures S2-S21 gather TEM micrographs and pictures of the materials synthesized.

As opposed to the case of $\mathrm{Pt}$, Ir, Ru or Pd, these experimental parameters have little influence on the resulting size and structure of the Os NPs. Methanol or ethanol are needed for the reaction to proceed and play the role of reducing agents. As opposed to the case of Pt, the synthesis is not influenced by the alcohol used, as confirmed by SAXS analysis, see Figure $\mathbf{S 2 2}$ and Table S2. Adding water leads to the formation of network-like structures reported in Figures S2-18, although the exact nature of this network is not yet established. The networks formed in the presence of water are not observed in the synthesis of $\mathrm{Pt}$, Ir or Ru NPs [22, 26, 29]. As opposed to Pt and Ir NP syntheses, Os NP synthesis proceed without the need for a base, Figures S18, S20-21. To develop a simple and relatively cheap synthesis of Os NPs requiring only few chemicals, it was concluded that $\mathrm{OsCl}_{3}$, no base and relatively high water content around 6075 v.\% using methanol as reducing agent were favourable conditions to obtain 
Os NPs for further studies. The Os NPs forming are in all cases ca. 1-2 nm, see TEM in Figure 1.
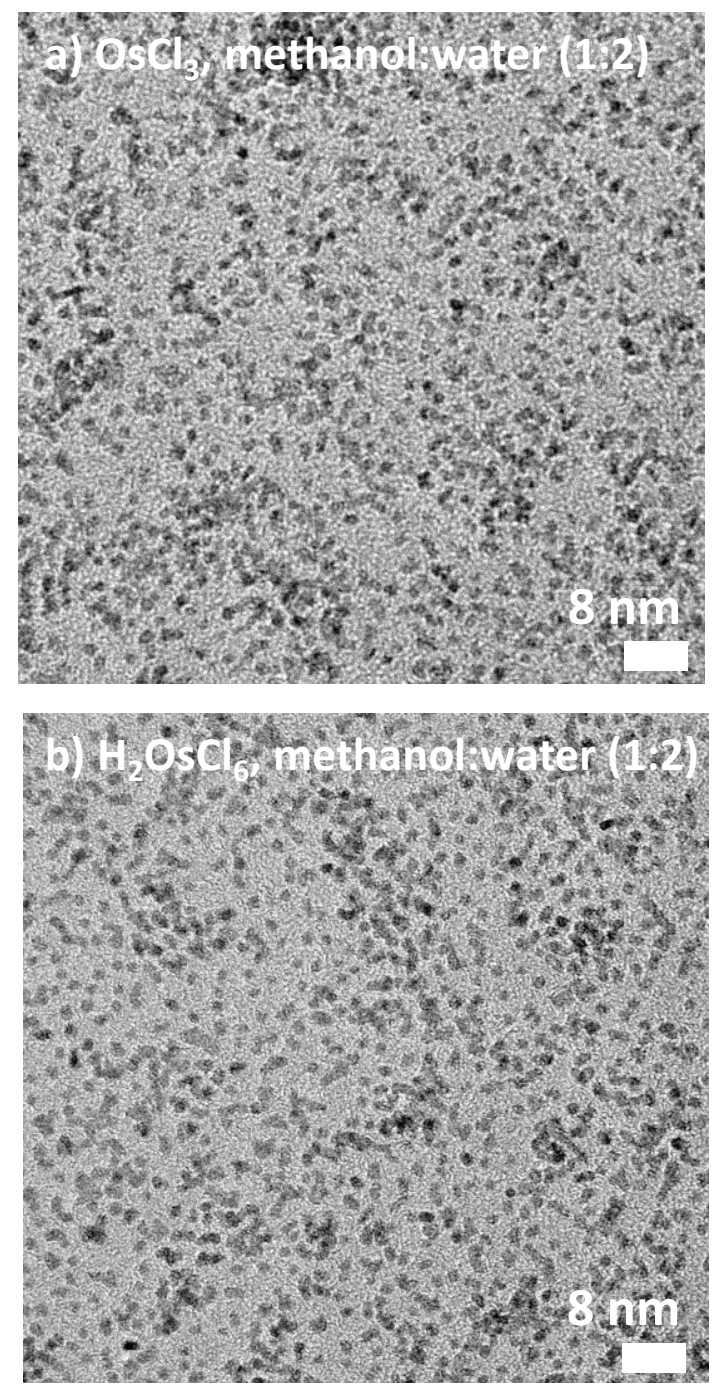

Figure 1: TEM micrographs of Os NPs obtained using 66 v. $\%$ water and 33 v. $\%$ methanol (no base) and $100 \mathrm{mM}$ (a) $\mathrm{OsCl}_{3}$ and (b) $\mathrm{H}_{2} \mathrm{OsCl}_{6}$ as precursor after 1 week reaction at $85^{\circ} \mathrm{C}$ in NMR tubes (volume ca. $0.2 \mathrm{~mL}$ ). The size analysis suggest that the NPs are (a) $1.6 \pm 0.4 \mathrm{~nm}$, (b) $1.7 \pm 0.3 \mathrm{~nm}$. 
Due to the small NP size, we turned to X-ray total scattering and pair distribution function analysis (PDF) to analyse the atomic structure of the Os NPs [33]. The total scattering signal is shown in Figure S25 and the resulting PDFs in Figure 2. The PDFs of the 3 samples are more or less identical, which is in line with the parameter study that these parameters do not influence the formed NPs. From the extent of features in the PDFs, it can be concluded that the NPs are all smaller than $2 \mathrm{~nm}$ with an average crystallite size between 1 and $2 \mathrm{~nm}$ which is in agreement with TEM and SAXS characterisation.

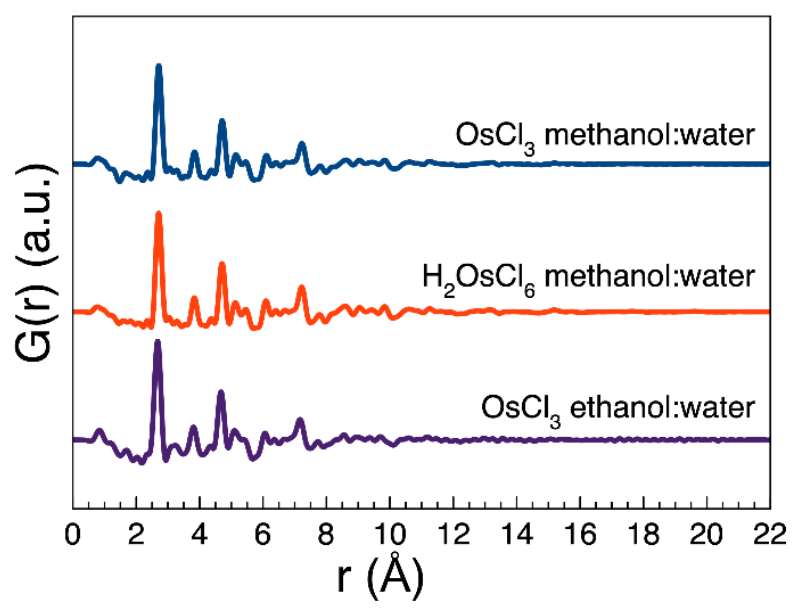

Figure 2: PDFs obtained from 4 different synthesis of Os NPs in 1:2 alcohol:water ratios and for different precursors as indicated. The PDFs can be described using a hcp model.

On the nanoscale, metallic NPs can take different structures that those of bulk metals, and icosahedral or decahedral motifs are often observed for metals with normally closed-packed structures [34]. By using the atomic simulation 
environment (ASE) module [35] and the cluster-mining approach developed by Banerejee et al. [34] we screened a large number of metal NP clusters, including $h c p, f c c$ and bcc, but also structures such as icosahedrons and decahedrons.

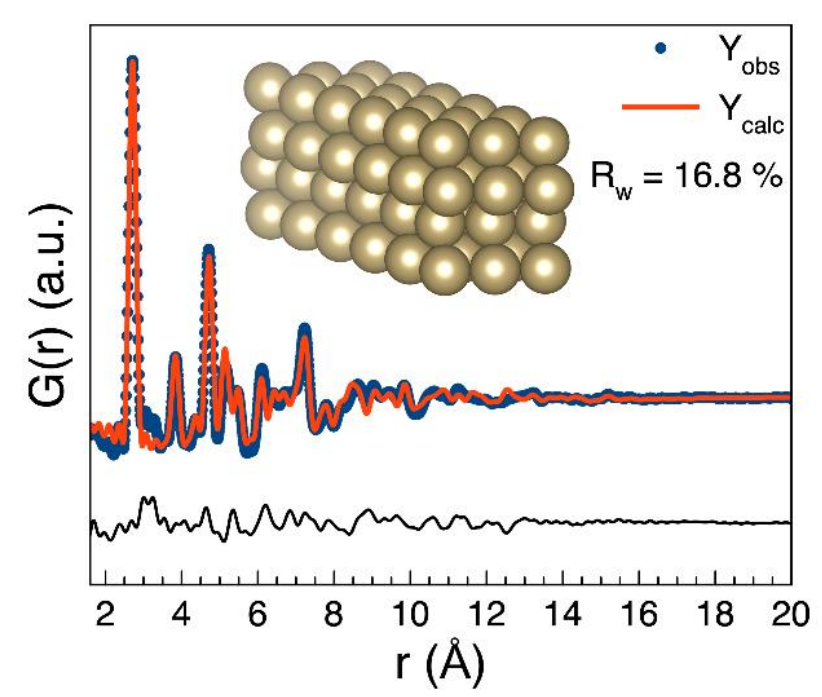

Figure 3: Fit of a $h c p$ cluster (seen in the insert) to the PDF obtained from the Os NPs formed in methanol:water from $\mathrm{OsCl}_{3}$.

The synthesised Os NPs are best described using a small $h c p$ cluster as seen in Figure 3 and Figures S26-31, which agrees with the bulk $h c p$ Os structure. As seen in Figure S39 the PDFs from the Os NPs synthesised from $\mathrm{OsCl}_{3}$ in ethanol:water show a small peak corresponding to an interatomic distance at 3.2 $\AA$. A small indication of the same peak may be seen as a shoulder for the two samples synthesized in methanol. The distance is not described in the $h c p$ model. We considered if this peak could originate from a ligand binding to the surface of the NPs, however, $3.2 \AA$ is significantly longer than Os-O, Os- $\mathrm{C}$ and 
Os-Cl bond distances which are ca. 1.8, $1.9 \AA$ and $2.3 \AA$, respectively. However, this peak at $3.2 \AA$ could correspond to an Os-Os distance in a [Os $\left.{ }_{2} \mathrm{Cl}_{2}\right]$ complex which may be present in the solution, see SI. A PDF from NPs synthesised from $\mathrm{H}_{2} \mathrm{OsCl}_{6}$ in ethanol:water was also obtained and is shown in Figure S40. As discussed in the SI, this PDF shows that the NPs synthesised from $\mathrm{H}_{2} \mathrm{OsCl}_{6}$ in ethanol:water have oxidised and so while the NPs remain mainly non-oxidised in solution they can oxidise over time or upon drying to form $\mathrm{OsO}_{4}$.

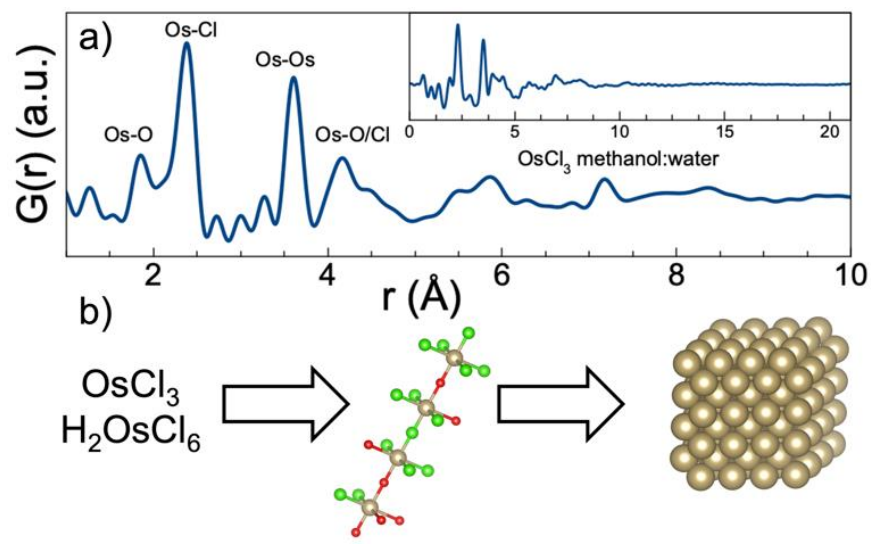

Figure 4: (a) Measured PDF of $\mathrm{OsCl}_{3}$ in methanol:water. The insert shows the same PDF plotted to $21 \AA$. (b) Overall formation mechanism of the hcp Os NPs. The Os chloride precursor reacts with the alcohol:water mixture to form chain-like structures of $\left[\mathrm{OsO}_{x} \mathrm{Cly}_{y}\right]$-octahedra which after long incubation time forms Os NPs. Os, $\mathrm{Cl}$ and $\mathrm{O}$ atoms shown in grey, green and red, respectively.

Total scattering experiments on the precursor solution were also performed, see Figure 4 and Figures S41-45. The resulting PDFs, are very similar to the PDFs of crystalline OsOCl compounds, e.g. OsOCl 2 and $\left(\mathrm{NH}_{4}\right)_{4}\left(\mathrm{OsOCl}_{10}\right)$, which form 
chain-like structures. Both structures are shown in Figures S43 and S44. This indicates that the precursors react with the solvent and form a network of chainlike structures of $\left[\mathrm{OsO}_{\mathrm{y}} \mathrm{Cl}_{\mathrm{x}}\right]$-octahedra, as illustrated in Figure 4. Note that the $\mathrm{O}$ atoms could be from a methoxy or ethoxy groups although PDF does not allow us to firmly conclude at this stage. The network is quite stable and even after 6 hours at $85^{\circ} \mathrm{C}$, minimal changes in the PDF occur for this high concentration of precursor $(100 \mathrm{mM})$ syntheses, see Figure S46. This stresses the need for longer syntheses time to initiate the breakdown of the precursor at high precursor concentration. This observation is in line with recent report on a formation mechanism involving chain-like structure made for the synthesis of Pt NPs [33]. In conclusion, Os NPs with a hcp crystal structure and a size around 1-2 nm are synthesized in methanol and ethanol and water mixtures from $\mathrm{OsCl}_{3}$ or $\mathrm{H}_{2} \mathrm{OsCl}_{6}$ precursors, without the need for surfactants. As opposed to the synthesis of Pt, Ir, Ru or Pd NPs by a similar approach, no base is here required. A synthesis using methanol:water in the volume ratio (1:2) with $\mathrm{OsCl}_{3}$ as precursor was here considered optimal. The results presented show that size control of Os NPs is a challenging task and even at high precursor concentration up to $100 \mathrm{mM}$ small size 1-2 nm in diameter NPs are obtained. X-ray total scattering measurement with PDF analysis show that the samples take the hcp structure, and it is shown that the Os NPs from from $\left[\mathrm{OsO}_{x} \mathrm{Cl}_{y}\right]$-complexes. This study and the versatile synthesis introduced provide a suitable platform to inspire future study of the formation mechanism of Os based nanomaterials and explore their properties further. 


\section{Supporting Information}

Supporting information file 1 (PDF): Supporting information for Surfactant-free syntheses and pair distribution function analysis of osmium nanoparticles, comprising:

Figure S1: Survey of precious metal literature

Figures S2-S21: TEM characterization and pictures of the nanomaterials obtained

Figure S22: SAXS characterization of the nanomaterials obtained

Figure S23: TEM characterization of the nanomaterials obtained

Figure S24: XRD characterization of the nanomaterials obtained

Figures S25-42: PDF characterization of the nanomaterials obtained

Figures S43-45: Crystal structures and models of the different clusters and complexes

Figure S46: PDF characterization of the nanomaterials obtained

Table S1: Overview of the parameters studied

Table S2: Fits parameters for SAXS

Tables S3-S13: Refined parameters for PDF

\section{Acknowledgements}

J. J. K Kirkensgaard and the Niels Bohr institute, University of Copenhagen, are thanked for access to SAXS.

\section{Funding}

JQ acknowledges the European Union's Horizon 2020 research and innovation program under the Marie Skłodowska-Curie grant agreement No. 840523 (CoSolCat). KMØJ is grateful to the Villum Foundation for financial support 
through a Villum Young Investigator grant (VKR00015416). This work was supported by the Danish National Research Foundation (DNRF-149) Center for High-Entropy Alloys Catalysis (CHEAC). This research used resources of the Advanced Photon Source, a U.S. Department of Energy (DOE) Office of Science User Facility, operated for the DOE Office of Science by Argonne National Laboratory under Contract No. DE-AC02-06CH11357 (GUP-72059).

\section{References}

1. Azharuddin, M.; Zhu, G. H.; Das, D.; Ozgur, E.; Uzun, L.; Turner, A. P. F.; Patra, H. K. Chem. Comm. 2019, 55 (49), 6964-6996.

2. Losch, P.; Huang, W. X.; Goodman, E. D.; Wrasman, C. J.; Holm, A.; Riscoe, A. R.; Schwalbe, J. A.; Cargnello, M. Nano Today 2019, 24, 15-47.

3. Chakraborty, I.; Pradeep, T. Chem. Rev. 2017, 117 (12), 8208-8271.

4. Hamalainen, J.; Sajavaara, T.; Puukilainen, E.; Ritala, M.; Leskela, M. Chem. Mater. 2012, 24 (1), 55-60.

5. Wakisaka, T.; Kusada, K.; Yamamoto, T.; Toriyama, T.; Matsumura, S.; Ibrahima, G.; Seo, O.; Kim, J.; Hiroi, S.; Sakata, O.; Kawaguchi, S.;

Kubota, Y.; Kitagawa, H. Chem. Commun. 2020, 56 (3), 372-374.

6. Adams, R. D.; Luo, Z. W. J. Organomet. Chem. 2016, 812, 108-114.

7. Han, L.; Wang, P. F.; Liu, H.; Tan, Q. Q.; Yang, J. J. Mater. Chem. A 2016, 4 (47), 18354-18365.

8. Kraemer, J.; Redel, E.; Thomann, R.; Janiak, C. Organometallics 2008, 27 (9), 1976-1978. 
9. Pitto-Barry, A.; Geraki, K.; Horbury, M. D.; Stavros, V. G.; Mosselmans, J. F. W.; Walton, R. I.; Sadler, P. J.; Barry, N. P. E. Chem. Commun. 2017, 53 (96), 12898-12901.

10. Danilovic, N.; Subbaraman, R.; Chang, K.-C.; Chang, S. H.; Kang, Y. J.;

Snyder, J.; Paulikas, A. P.; Strmcnik, D.; Kim, Y.-T.; Myers, D.;

Stamenkovic, V. R.; Markovic, N. M. J. Phys. Chem. Lett. 2014, 5 (14), 2474-2478.

11. Anantharaj, S.; Nithiyanantham, U.; Ede, S. R.; Kundu, S. Ind. Eng. Chem. Res. 2014, 53 (49), 19228-19238.

12. Metin, O.; Alp, N. A.; Akbayrak, S.; Bicer, A.; Gultekin, M. S.; Ozkar, S.;

Bozkaya, U. Green Chem. 2012, 14 (5), 1488-1492.

13. Egeberg, A.; Dietrich, C.; Kind, C.; Popescu, R.; Gerthsen, D.; Behrens, S.; Feldmann, C. ChemCatChem 2017, 9 (18), 3534-3543.

14. Lim, C. S.; Sofer, Z.; Toh, R. J.; Eng, A. Y. S.; Luxa, J.; Pumera, M. ChemPhysChem 2015, 16 (9), 1898-1905.

15. Sanchez-Cano, C.; Gianolio, D.; Romero-Canelon, I.; Tucoulou, R.; Sadler, P. J. Chem. Commun. 2019, 55 (49), 7065-7068.

16. Odularu, A. T.; Ajibade, P. A.; Mbese, J. Z.; Oyedeji, O. O. J. Chem. 2019, 2019, ID 5459461.

17. He, S. B.; Yang, L.; Balasubramanian, P.; Li, S. J.; Peng, H. P.; Kuang, Y.; Deng, H. H.; Chen, W. J. Mater. Chem. A 2020, 8 (47), 25226-25234.

18. Escano, M. C. S.; Arevalo, R. L.; Gyenge, E.; Kasai, H. Catal. Sci. Technol. 2014, 4 (5), 1301-1312. 
19. Niu, Z. Q.; Li, Y. D. Chem. Mater. 2014, 26 (1), 72-83.

20. Nithiyanantham, U.; Ede, S. R.; Kundu, S. J. Mater. Chem. C 2014, 2 (19), 3782-3794.

21. Vollmer, C.; Redel, E.; Abu-Shandi, K.; Thomann, R.; Manyar, H.; Hardacre, C.; Janiak, C. Chem. Eur. J. 2010, 16 (12), 3849-3858.

22. Quinson, J.; Neumann, S.; Wannmacher, T.; Kacenauskaite, L.; Inaba, M.; Bucher, J.; Bizzotto, F.; Simonsen, S. B.; Kuhn, L. T.; Bujak, D.; Zana, A.; Arenz, M.; Kunz, S. Angew. Chem. 2018, 130 (38) 12518-12521; Angew. Chem. Int. Ed. 2018, 57 (38), 12338-12341.

23. Quinson, J.; Kunz, S.; Arenz, M. ChemCatChem 2021, 13 (7), 1692-1705.

24. Quinson, J. Front. Nanotechnol. 2021, 3, ID 770281.

25. Quinson, J.; Neumann, S.; Kacenauskaite, L.; Bucher, J.; Kirkensgaard, J. J. K.; Simonsen, S. B.; Kuhn, L. T.; Zana, A.; Vosch, T.; Oezaslan, M.; Kunz, S.; Arenz, M. Chem. Eur. J. 2020, 26 (41), 9012-9023.

26. Quinson, J.; Kacenauskaite, L.; Bucher, J.; Simonsen, S. B.; Kuhn, L. T.; Oezaslan, M.; Kunz, S.; Arenz, M. ChemSusChem 2019, 12 (6), 12291239.

27. Quinson, J.; Simonsen, S. B.; Kuhn, L. T.; Kunz, S.; Arenz, M. RSC Adv. 2018, 8 (59), 33794-33797.

28. Bizzotto, F.; Quinson, J.; Schröder, J.; Zana, A.; Arenz, M. J. Catal. 2021, $401,54-62$.

29. Bizzotto, F.; Quinson, J.; Zana, A.; Kirkensaard, J. J. K.; Dworzak, A.; Oezaslan, M.; Arenz, M. Catal. Sci. Technol. 2019, 9 (22), 6345-6356. 
30. Quinson, J.; Bucher, J.; Simonsen, S. B.; Kuhn, L. T.; Kunz, S.; Arenz, M. ACS Sustain. Chem. Eng. 2019, 7 (16), 13680-13686.

31. Quinson, J.; Kacenauskaite, L.; Schroder, J.; Simonsen, S. B.; Kuhn, L. T.; Vosch, T.; Arenz, M. Nanoscale Adv. 2020, 2 (6), 2288-2292.

32. Quinson, J.; Jensen, K. M. Ø. Adv. Colloid Interface Sci. 2020, 286, 102300.

33. Mathiesen, J. K.; Quinson, J.; Dworzak, A.; Vosch, T.; Juelsholt, M.; Kjaer, E. T. S.; Schroder, J.; Kirkensgaard, J. J. K.; Oezaslan, M.; Arenz, M.; Jensen, K. M. O. J. Phys. Chem. Lett. 2021, 12 (12), 3224-3231.

34. Banerjee, S.; Liu, C.-H.; Jensen, K. M. O.; Juhas, P.; Lee, J. D.; Tofanelli, M.; Ackerson, C. J.; Murray, C. B.; Billinge, S. J. L. Acta Cryst. A 2020, 76 (1), 24-31.

35. Hjorth Larsen, A.; Jørgen Mortensen, J.; Blomqvist, J.; Castelli, I. E.; Christensen, R.; Dułak, M.; Friis, J.; Groves, M. N.; Hammer, B.; Hargus, C.; Hermes, E. D.; Jennings, P. C.; Bjerre Jensen, P.; Kermode, J.; Kitchin, J. R.; Leonhard Kolsbjerg, E.; Kubal, J.; Kaasbjerg, K.; Lysgaard, S.; Bergmann Maronsson, J.; Maxson, T.; Olsen, T.; Pastewka, L.; Peterson, A.; Rostgaard, C.; Schiøtz, J.; Schütt, O.; Strange, M.; Thygesen, K. S.; Vegge, T.; Vilhelmsen, L.; Walter, M.; Zeng, Z.; Jacobsen, K. W. J. Phys.: Condens. Matter. 2017, 29 (27), 273002. 